\title{
Characterization of SNDR Degradation in Nonlinear Wireless Transmitters
}

\author{
Rui Estanqueiro Santos, ${ }^{1}$ Nuno Borges Carvalho,, ${ }^{1}$ Kevin Gard ${ }^{2}$ \\ ${ }^{1}$ Instituto de Telecomunicações, Universidade de Aveiro, Portugal \\ ${ }^{2}$ North Carolina State University, Raleigh, NC \\ Received 12 August 2008; accepted 28 November 2008
}

\begin{abstract}
This article presents a measurement strategy and a characterization bench for the evaluation of Signal to Noise and Distortion Ratio (SNDR) in nonlinear wireless systems when in presence of memory effects. This measurement proposal will be combined with a closed analysis of the nonlinear mechanisms appearing in communication systems presenting memory. The analysis of this memory mechanism, when the system is excited by complex modulated signals with different statistics, will be a useful tool for design engineers to evaluate the degradation of SNDR in nonlinear wireless systems. Finally, the proposed measurement approach will be applied to a CDMA communication system, by evaluating carefully the SNDR of that system. () 2009 Wiley Periodicals, Inc. Int J RF and Microwave CAE 19: 470-480, 2009.
\end{abstract}

Keywords: power amplifiers; nonlinear systems; long term memory

\section{INTRODUCTION}

The main cause of SNDR degradation in wireless transmitters is the nonlinear behavior of the power amplifier (PA) output stage. This nonlinear behavior can be seen at the output signal as adjacent channel distortion, which was already studied, mainly by analyzing the spectral regrowth of the output signals, (e.g., studying adjacent channel power ratio, ACPR), or can be seen as cochannel distortion, normally studied by analyzing figures of merit like noise power ratio (NPR) or error vector magnitude (EVM).

Nevertheless and due to the fact that cochannel distortion is precisely coincident with the output linear signal, it is very difficult to characterize

Correspondence to: N. B. Carvalho; e-mail: nbcarvalho@ua.pt DOI 10.1002/mmce. 20370

Published online 13 April 2009 in Wiley InterScience (www. interscience.wiley.com). and measure. Moreover, most of the measurement techniques are based exclusively on approaches applied to memoryless nonlinearities. ${ }^{1}$ Unfortunately, and mainly due to the increase in system bandwidth, this memoryless assumption is no longer valid, and most of the used techniques are becoming obsolete.

Some of these no memoryless manifestations are the so called long-term memory effects, MEs, which are recognized as an important problem in wideband wireless communication systems.

The impact of this type of phenomena is responsible for limiting the effectiveness of linearizing techniques, and the degradation in EVM values compared with the ones usually obtained with memoryless systems.

\footnotetext{
${ }^{1}$ By memoryless devices we consider nonlinear devices in which the output signal changes instantaneously with the input signal.
} 
This degradation can be accounted by calculating the Signal to Noise and Distortion Ratio, SNDR [1], defined as [2]:

$$
\begin{aligned}
\operatorname{SNDR}(\omega) & =\frac{S_{\mathrm{o}}(\omega)}{N(\omega)+D(\omega)} \\
& =\frac{G_{\mathrm{cor}}^{2} \cdot S_{\mathrm{i}}(\omega)}{G_{\mathrm{cor}}^{2} \cdot N_{\mathrm{i}}(\omega)+N_{\mathrm{a}}(\omega)+D(\omega)}
\end{aligned}
$$

where the $G_{\mathrm{cor}}$ is the so called underlying linear gain, that corresponds to the linear power gain of the system, $N_{\mathrm{i}}$ and $S_{\mathrm{i}}$ are the input noise and input signal power spectral densities correspondingly, $N_{\mathrm{a}}$ is the power density of the PA addictive noise and $D$ is the power density of stochastic cochannel nonlinear distortion.

A carefully study of the cochannel nonlinear distortion is fundamental in order to determine a correct value of SNDR, and thus of the degradation of quality transmission in a wireless communication signal. Signal waveform quality is often defined by the well known EVM, which can be related to SNDR by [1]:

$$
\mathrm{EVM}=\sqrt{\frac{1}{\mathrm{SNDR}}}
$$

As the SNDR is imposed by the cochannel distortion, and that is due to the uncorrelated nonlinear contribution to the overall output signal, it is important to distinguish distortion components which are correlated and uncorrelated in respect to the input signal.

The main problem related to this aspect is that most of the authors that have addressed the problem of cochannel nonlinear distortion, have address it for memoryless nonlinear behavior [3-5], mainly due to the fact that the systems evaluated where low bandwidth, and analysis and modeling of memory effects were not well understood at the time.

Recently that previous mathematical approaches were expanded to systems presenting memory [6] with QPSK modulated signals.

In this article, we will address the degradation of SNDR in nonlinear transmitters when they present memory effects. The work is mainly devoted to present in a clear and definite way how to characterize and measure cochannel distortion in presence of nonlinear memory effects.

The main novelties of this article can thus be described as an explanation on how to measure cochannel distortion in nonlinear devices presenting memory, and on the impact of the memory effects in the cochannel distortion of real wireless transmitters.
The article will first start with a simple explanation of the model used to obtain the mathematical results, then, we will present a measurement bench to extract the cochannel distortion, and complement this analysis by presenting some simulated and measured data of a dynamic nonlinear wireless transmitter when excited by an IS-95 reverse link signal.

\section{COCHANNEL DISTORTION EVALUATION}

Cochannel distortion has been for many years a problem of concern due to the fact that it is very difficult to evaluate. The main reason for this is that the cochannel distortion falls exactly over the linear output signal, which is several dB's above the seek distortion, thus the separation of these two types of signal is quite difficult.

The traditional figure of merit for evaluating cochannel distortion is called noise power ratio, NPR [7], where a slice of the input signal is extracted and the noise is further measured in that notch, both at the input and output, the relationship between them, gives the increased degradation of the cochannel distortion. Nevertheless this figure of merit is not $100 \%$ exact, as was previously presented in $[8,9]$, where a new figure of merit was proposed, the cochannel power ratio, CCPR, and the cochannel interference ratio, CIR. In both of those cases the cochannel distortion was evaluated canceling the output linear components, case of CCPR [8], and the output correlated component case of CIR [9].

In the CCPR case the figure of merit accounts for every distortion at the output signal, both the correlated and uncorrelated cochannel distortion, which is very important for amplitude modulated signals, while in the CIR case the figure of merit accounts mainly for the uncorrelated distortion at the output.

By correlated distortion we mean the output components of the nonlinear distortion that are correlated with the input i.e., the part that obeys:

$$
R_{\mathrm{xy}}(\tau)=E\left\{y(t) x(t+\tau)^{*}\right\}=\int_{-\infty}^{\infty} y(t) x(t+\tau) d t
$$

This is called the cross-correlation between the output signal $y(t)$ and the input signal $x(t)$, where $E(.$.$) is$ the average over measured timed windows.

Using the theory proposed from Bendat [10], to extract the correlated and the uncorrelated distortions, we will start by calculating different values of cross- 
correlation functions for the input, output and crossvalues.

The output autocorrelation will be obtained as:

$$
R_{\mathrm{yy}}(\tau)=E\left\{y(t) y(t+\tau)^{*}\right\}=\int_{-\infty}^{\infty} y(t) y(t+\tau) d t
$$

where $R_{\mathrm{yy}}(\tau)$ is the output autocorrelation. It should be noticed that:

$$
\begin{aligned}
& R_{\mathrm{yy}}(\tau)=E\left[y(t) y^{*}(t+\tau)\right] \\
& \quad=E\left\{\left[y_{1}(t)+y_{\text {dist }}(t)\right]\left[y_{1}(t+\tau)+y_{\text {dist }}(t+\tau)\right]^{*}\right\} \\
& \quad=E\left\{\left[y_{1}(t)\right]\left[y_{1}(t+\tau)\right]^{*}\right\}+E\left\{\left[y_{1}(t)\right]\left[y_{\text {dist }}(t+\tau)\right]^{*}\right\} \\
& \quad+E\left\{\left[y_{\text {dist }}(t)\right]\left[y_{1}(t+\tau)\right]^{*}\right\}+E\left\{\left[y_{\text {dist }}(t)\right]\left[y_{\text {dist }}(t+\tau)\right]^{*}\right\}
\end{aligned}
$$

In expression (5) we are considering that the output signal can be decomposed as a linear component, $y_{1}(t)$, and a distorted components $y_{\text {dist }}(t)$, which is in fact the usual way well behaved nonlinearities work.

From (5) we can also see that the first term is the linear response, the second and third terms are the correlated part of the output signal (referring the input), ${ }^{2}$ and the forth term is the nonlinear distortion correlation, usually called in [3, 4] spectral regrowth, which unfortunately also has some correlated components with the linear part of the output.

This is in fact one of the major problems of nonlinear systems, that is, how to identify the different signal component at the output. One of the solutions often used is to consider as signal the output component which is correlated with the input, as is usual in conventional wireless rake receivers.

Bussgang's Theorem [2, 4] provides the theoretical support for this, since by correlating the output and input signals, we can estimate the effective signal component of the output, and thus by subtraction we can also obtain the uncorrelated part, which is in fact the output nonlinear distortion noise.

So, we can also divide the output into components which are correlated and uncorrelated in respect to the input signal, and rewrite (5) as:

$$
\begin{aligned}
& R_{\mathrm{yy}}(\tau)=E\left[y(t) y^{*}(t+\tau)\right] \\
& \quad=E\left\{\left[y_{\mathrm{c}}(t)+y_{\mathrm{u}}(t)\right]\left[y_{\mathrm{c}}(t+\tau)+y_{\mathrm{u}}(t+\tau)\right]^{*}\right\} \\
& \quad=E\left\{\left[y_{\mathrm{c}}(t)\right]\left[y_{\mathrm{c}}(t+\tau)\right]^{*}\right\}+E\left\{\left[y_{\mathrm{c}}(t)\right]\left[y_{\mathrm{u}}(t+\tau)\right]^{*}\right\} \\
& \quad+E\left\{\left[y_{\mathrm{u}}(t)\right]\left[y_{\mathrm{c}}(t+\tau)\right]^{*}\right\}+E\left\{\left[y_{\mathrm{u}}(t)\right]\left[y_{\mathrm{u}}(t+\tau)\right]^{*}\right\} \\
& \quad=E\left\{\left[y_{\mathrm{c}}(t)\right]\left[y_{\mathrm{c}}(t+\tau)\right]^{*}\right\}+E\left\{\left[y_{\mathrm{u}}(t)\right]\left[y_{\mathrm{u}}(t+\tau)\right]^{*}\right\}
\end{aligned}
$$

$y_{\mathrm{u}}$ and $y_{\mathrm{c}}$ are the correlated and uncorrelated components respectively.

\footnotetext{
${ }^{2}$ These terms are related to gain compression and expansion.
}

Combining (3) to (6) we found that the correlated part of the output could be given by:

$$
\begin{aligned}
R_{y_{\mathrm{c}} y_{\mathrm{c}}}(\tau)=\left|G_{\mathrm{cor}}\right|^{2} E\left\{x(t) x(t+\tau)^{*}\right\} \\
=\left|G_{l}+G_{\text {distc }}\right|^{2} E\left\{x(t) x(t+\tau)^{*}\right\}
\end{aligned}
$$

where $G_{\text {cor }}$ is called the underlying linear system gain, $G_{1}$ the linear gain and $G_{\text {distc }}$ the correlated distortion gain which is defined by cross correlation between the distorted output signal and the input signal, which is given by:

$$
G_{\text {distc }}=\frac{E\left\{\left[y_{\text {dist }}(t)\right][x(t+\tau)]^{*}\right\}}{E\left\{[x(t)][x(t+\tau)]^{*}\right\}}
$$

The uncorrelated component distortion is defined by subtracting the correlated component from the output, ${ }^{3}$ thus:

$$
\begin{aligned}
& R_{y_{\mathrm{u}} y_{\mathrm{u}}}(\tau)=E\left\{\left[y_{\mathrm{dist}}(t)\right]\left[y_{\mathrm{dist}}(t+\tau)\right]^{*}\right\} \\
&-\left|G_{\mathrm{distc}}\right|^{2} E\left\{[x(t)][x(t+\tau)]^{*}\right\}
\end{aligned}
$$

The underlying linear system gain is given by:

$$
G_{\mathrm{cor}}=\frac{E\left\{y(t) x(t+\tau)^{*}\right\}}{E\left\{x(t) x(t+\tau)^{*}\right\}}
$$

After these calculations the system SNDR will thus be:

$$
\begin{aligned}
\operatorname{SNDR}(\omega) & =\frac{S_{\mathrm{o}}(\omega)}{N(\omega)+D(\omega)} \\
& =\frac{G_{\mathrm{cor}} S_{i}(\omega)}{G_{\mathrm{cor}} N_{\mathrm{i}}(\omega)+N_{\mathrm{a}}(\omega)+\Im\left[R_{\mathrm{y}_{\mathrm{u}} y_{\mathrm{u}}}(\tau)\right]}
\end{aligned}
$$

where $\mathfrak{I}\left[R_{\mathrm{y}_{\mathrm{u}} \mathrm{y}_{\mathrm{u}}}(\tau)\right]$ is the Fourier transform of the uncorrelated output autocorrelation function and $N_{\mathrm{a}}(\omega)$ is the added noise of the transmitter.

\section{COCHANNEL DISTORTION MEASUREMENT BENCH}

As was seen in section II, the uncorrelated distortion could be obtained by using expression (9), which is

\footnotetext{
${ }^{3}$ It should be noted that in (9) we have used exclusively the distorted components for evaluating the uncorrelated distortion. However, in the real measured results we do not have direct access to just the distorted signal, because it is combined with the linear output signal, thus the overall underlying linear system gain should be used. This approach degrades the overall dynamic range of the measurements.
} 


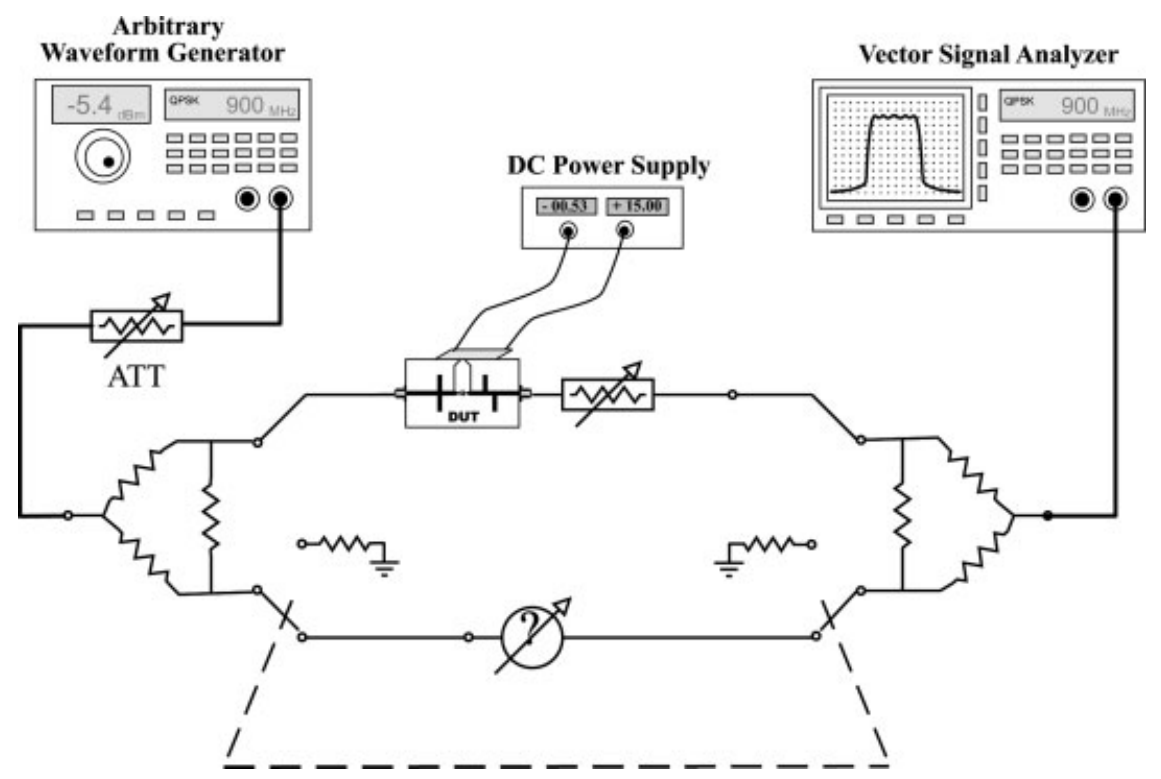

Figure 1. Feed forward cancellation technique.

an extreme complex analysis if made in the time domain, so the usual way to do it is by first calculate the spectrum at the output and input ports, and then the corresponding underlying linear system gain in the frequency domain. Thus the output uncorrelated spectrum will be:

$$
S_{y_{\mathrm{u}} y_{\mathrm{u}}}(\omega)=S_{\mathrm{yy}}(\omega)-\left|G_{\mathrm{cor}}(\omega)\right|^{2} S_{\mathrm{xx}}(\omega)
$$

where $S_{\mathrm{y}_{\mathrm{u}} \mathrm{y}_{\mathrm{u}}}(\omega)$ and $S_{\mathrm{xx}}(\omega)$ are the power spectrum densities of the output uncorrelated distortion and the input signal respectively.

This way the output uncorrelated spectra is directly obtained.

Nevertheless this formulation depends on a cancellation between the output signal spectra and the output equivalent correlated contribution spectra, thus suffering from numerical error arising from the cancellation procedure.

Actually the calculation of the cross-correlation terms is very intensive, and suffers from a high degree of measurement noise, since several slices of the time domain modulated signal should be acquired before the calculations are done.

Moreover the number of random windows to use is extremely high for a correct identification of the nonlinear cochannel distortion; this is the reason why most authors that have dealt with this problem have selected an alternative measurement scheme.

The alternative solution is to implement the cancellation of the correlated part in the instrumentation itself, rather than in a numerical computer.
One proposal for an instrumentation of that kind, is based on the application of a feed-forward cancellation loop as presented in [8] and [9], Figure 1.

The output of this loop is the subtraction of the overall output signal (including the distortion), with a copy of the linear output component.

The first path corresponds to the overall output signal, while the second path is an approximation of the underlying linear gain of the DUT.

The traditional approaches tune the loop either at the small signal excursion, where the output is mainly a linear replica of the input, case of CCPR, and thus all the output distortion is measured, or the loop is tuned at the operational power, case of CIR, where the output uncorrelated distortion is measured.

However these approaches were obtained for memoryless nonlinear components, where the feedforward path could correctly approximate the underlying linear gain of the system, ${ }^{4}$ as was presented in $[3,8,9]$.

Unfortunately in the case of PA having a large impact of memory effects, that is not correct, as the underlying linear system is quite different from a linear well behaved gain [2], and so by applying this feed-forward scheme the output will continue to have a certain amount of correlated distortion that could not be approximated by the delay plus attenuator approach.

${ }^{4}$ In memoryless systems, the underlying system gain is normally approached by an attenuator followed by a phase shifter. 
To better understand this fact, consider a memoryless nonlinearity and a nonlinearity presenting memory.

\section{A. Memoryless Nonlinearity}

In a simple memoryless nonlinear component the inband signal can be roughly approximated by [7]:

$$
y(t)=a_{1} \cdot x(t)+a_{3} \cdot x^{3}(t)+\cdots
$$

where we consider that the nonlinearity can be approximated by a simple third order polynomial, and $a_{1}$ and $a_{3}$ are the polynomial coefficients.

In this case the in-band distorted output can be approximated by a nonlinear transfer function that is given by:

$$
H_{3}\left(\omega_{x}, \omega_{y},-\omega_{z}\right)=K_{3}
$$

where $H_{3}(\ldots)$ is the nonlinear operator in the sense of Volterra Series [7] and $K_{3}$ is constant over the bandwidth of interest for memoryless devices.

This states that the feed-forward second path in Figure 1, can be approximated by a simple attenuator and a phase shifter, as has been the traditional way to do it.

The output of the loop will thus be:

$$
S_{\text {yyloop }}(\omega)=S_{\text {yy }}(\omega)-\left|G_{\text {cor }}(\omega)\right|^{2} S_{\mathrm{xx}}(\omega)
$$

where $S_{\text {yyloop }}(\omega)$ is the output of the loop power spectral density.

But since the correlated gain was perfectly modeled, and do not change with frequency, then:

$$
S_{\text {yyloop }}(\omega)=S_{y_{\text {unc }} y_{\text {unc }}}(\omega)
$$

Thus the measured valued is a good approximation of the uncorrelated component power spectral density.

\section{B. Nonlinearity Presenting Memory}

In the case where the nonlinearity presents memory, the signal behavior can be approximated by a feedback mechanism that imposes nonlinear distortion inside the band of interest, by somehow up-converting the low frequency behavior of the transmitter to the in-band RF frequency. A deep study of this type of scheme can be found in $[11,12]$.

Some authors have represented these effects using some special forms of behavioral models [13], which somehow represent the mechanism that were already

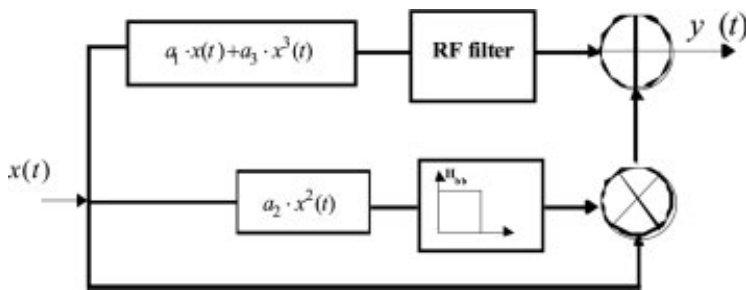

Figure 2. Proposed Behavioral model with long-term memory effects.

described. The proposed model from [13] is presented in Figure 2.

The output of this model is given by ${ }^{5}$ :

$$
\begin{aligned}
y(t) & =a_{1} \cdot x(t)+a_{2} \cdot x(t) \\
\cdot & {\left[\int_{-\infty}^{+\infty} x^{2}(t-\tau) \cdot h_{\mathrm{bb}}(\tau) d \tau\right]+a_{3} \cdot x^{3}(t) }
\end{aligned}
$$

where $a_{2}$ is the second order polynomial coefficient and $h_{\mathrm{bb}}(\tau)$ is the impulse response of the base band filter.

When we compare this model with a memoryless nonlinearity the new approach includes a filtered second-order interaction term that is responsible for the up-conversion of the low frequency behavior of the transmitter.

Based on the work presented in [12] it is known that the fundamental zone nonlinear distortion can be described by ${ }^{6}$ :

$$
\begin{aligned}
H_{3}\left(\omega_{x}, \omega_{y},-\omega_{z}\right)=K_{3}-F_{2}\left(\omega_{x},\right. & \left.-\omega_{z}\right) \\
& -F_{2}\left(\omega_{y},-\omega_{z}\right)
\end{aligned}
$$

where $F_{2}($.) is a nonlinear operator representing the second-order interaction component which is frequency dependent.

An implication that can be seen here is that most of the spectrum shape that appears at the cochannel and adjacent channel are tailored by the response of the baseband filter.

Thus it is expected that the memory behavior will affect both the correlated and uncorrelated spectrum at the output. In [12] it was also proved that the cor-

\footnotetext{
${ }^{5}$ In this case, we consider that the impact of the RF filter can be ignored.

${ }^{6}$ Similar assumptions as used in [12], where also considered here, that is: the second harmonic filter and the in-band filter are considered flat all over the interesting bandwidth.
} 
related part, in respect to the input signal, is only affected by terms equal to:

$$
H_{3}\left(\omega_{x}, \omega_{y},-\omega_{y}\right)=K_{3}-F_{2}(0)-F_{2}\left(\omega_{x},-\omega_{y}\right)
$$

where $F_{2}(0)$ is the equivalent second order function evaluated at $0 \mathrm{~Hz}$, or DC, thus memoryless, since it does not change with frequency, and only the term $F_{2}$ $\left(\omega_{x},-\omega_{y}\right)$ is responsible for the memory behavior.

When describing the uncorrelated part we have the summation of two different $F_{2}()$ terms as in (18) that will contribute to the shape of the in-band spectrum.

Thus if we have a transmitter presenting memory excited by a complex modulated signal its output spectrum characteristics will be deeply impacted by the memory, and can even change significantly compared to its memoryless behavior.

Because of the feed-forward loop consists only on a simple attenuator and a phase shifter, the output of the loop in this case will be:

$$
S_{\text {yyloop }}(\omega)=S_{y y}(\omega)-\left|G_{\text {memorylesscorr }}(\omega)\right|^{2} S_{x x}(\omega)
$$

where $G_{\text {memorylesscorr }}(\omega)$ is the underlying linear system gain that includes the pure linear gain and the correlated gain arising from the memoryless nonlinear distortion part, that can be efficiently represented by an attenuator followed by a phase shifter.

Looking at expression (7) the correlated gain will then be divided into a pure linear part, a correlated linear gain coming out from the memoryless distortion and a component of the correlated memory distortion, as in expression (22).

$\left|G_{\text {cor }}\right|^{2}=\left|G_{l}+G_{\text {distc }}\right|^{2}=\left|G_{\text {memorylesscorr }}+G_{\text {memorycorr }}\right|^{2}$

where $G_{\text {memorylesscorr }}$ and $G_{\text {memorycorr }}$ are the underlying linear gain of the memoryless and the memory components respectively.

These gains will be added as they are all correlated to each other.

As we can assume that the second path of the feed-forward loop will approximate the memoryless underlying linear gain with good confidence, which means that part of the correlated distortion will be subtracted from the output, the uncorrelated distortion at the loop output will then be:

$$
S_{y_{\mathrm{u}} y_{\mathrm{u}}}(\omega)=S_{\text {yyloop }}(\omega)-\left|G_{\text {memorycorr }}(\omega)\right|^{2} S_{\mathrm{xx}}(\omega)
$$

In this scenario the amount of correlated signal at the output is now much more reduced, and thus the numerical error will be further diminished, as already stated for the simulation case, where uncorrelated distortion is normally calculated directly over the distortion components.

The value of $\left|G_{\text {memorycorr }}(\omega)\right|^{2}$ can be directly calculated from:

$$
G_{\text {memorycorr }}(\tau)=\frac{E\left\{\left[y_{\text {loop }}(t)\right][x(t+\tau)]^{*}\right\}}{E\left\{[x(t)][x(t+\tau)]^{*}\right\}}
$$

where $y_{\text {loop }}(t)$ is the signal at the output of the loop, and in the frequency domain as:

$$
G_{\text {memorycorr }}(\omega)=\frac{S_{\text {yloopx }}(\omega)}{S_{\mathrm{xx}}(\omega)}
$$

where $S_{\text {yloopx }}(\omega)$ is the power spectral density corresponding to the cross correlation of the output of the loop and the input signal.

This formulation defines the measurement procedure to be used in the evaluation of cochannel distortion in the situation of nonlinear transmitters presenting memory effects.

As was seen, the main difference resides in the fact that the signal is acquired at the output of the loop, after the cancelation of the memoryless correlated distortion.

Then the uncorrelated distortion is calculated in a computer machine, reducing this way the numerical error.

\section{COCHANNEL DISTORTION CHARACTERIZATION IN A REAL CDMA SYSTEM}

In this section the previous proposed theory will be applied to a wireless transmitter when excited by a modulated signal. An evaluation of SNDR will be done, either by simulation and measurement results, of a memoryless transmitter and a transmitter presenting memory.

The transmitter is composed of an O-QPSK modulator followed by a PA, which is modeled accordingly to the model presented on Figure 2.

The modulator system architecture is presented on Figure 3.

The input signal for this modulator can be described as:

$$
x(t)=I(t) \cos \left(\omega_{\mathrm{c}} t+\theta\right)+Q(t) \sin \left(\omega_{\mathrm{c}} t+\theta\right)
$$




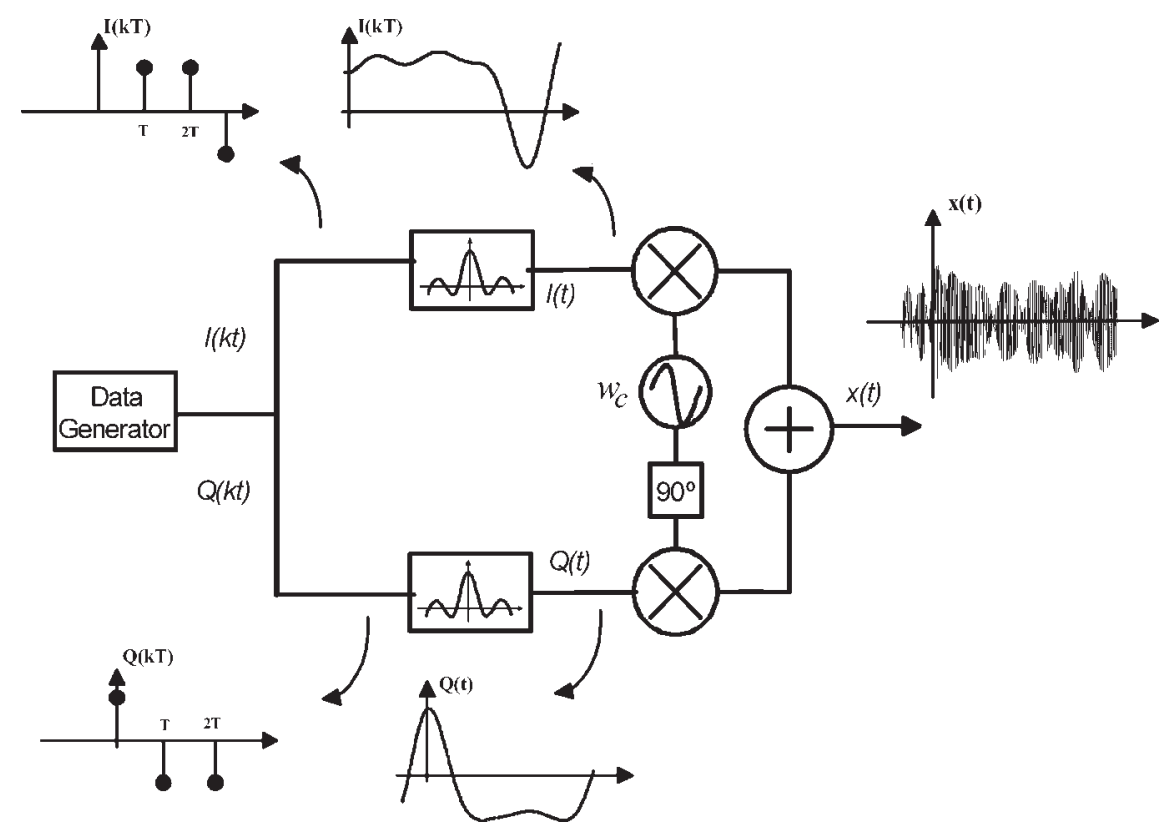

Figure 3. I/Q QPSK modulator.

Where the $I$ and $Q$ components are:

$$
\begin{gathered}
I(t)=\sum_{k=-\infty}^{+\infty} i_{\mathrm{k}} h(B t-k) \\
Q(t)=\sum_{k=-\infty}^{+\infty} q_{\mathrm{k}} h\left(B t-k+\frac{1}{2}\right)
\end{gathered}
$$

with $i_{\mathrm{k}}$ and $q_{\mathrm{k}}$ the dirac delta functions representing the split binary information into $\mathrm{I}$ and $\mathrm{Q}$ branches respectively, $h(\ldots)$ is the pulse shaping filter, $\omega_{\mathrm{c}}$ is the angular frequency of the carrier and $\theta$ a random phase.

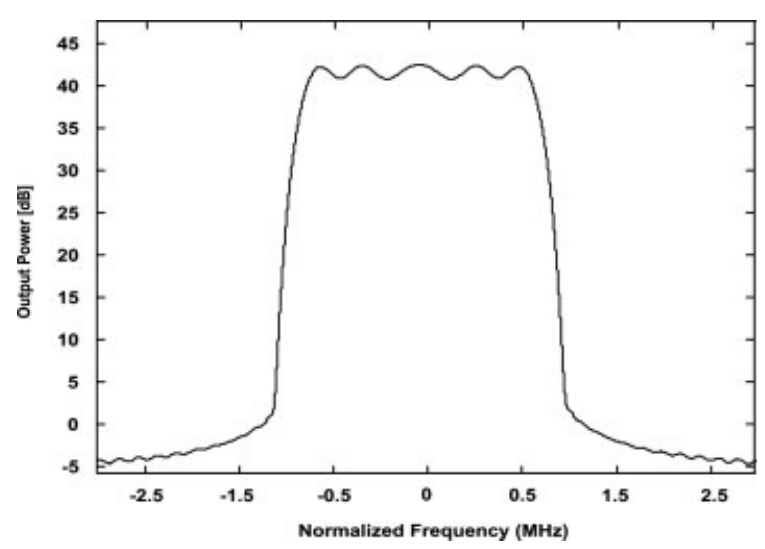

Figure 4. RF wireless spectrum.
The output spectrum of the modulated wireless RF signal can be seen in Figure 4.

This signal will further excite a nonlinear PA. When the PA presents no memory, the output spectrum is presented on Figure 5, where the cochannel distortion is clearly seen, this result is perfectly equal to the results presented by Aparin in [3].

A memory path was then inserted into the PA model, as presented on Figure 2, representing the amplifier memory effects, and different values of the base-band filter were used.

The total output spectrum mask, including the correlated and uncorrelated part, is presented on

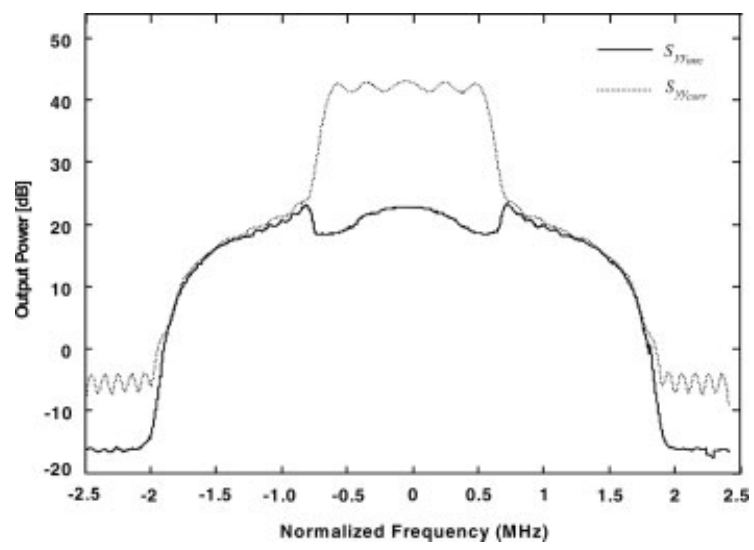

Figure 5. Total memoryless correlated and uncorrelated output spectrum shape. 


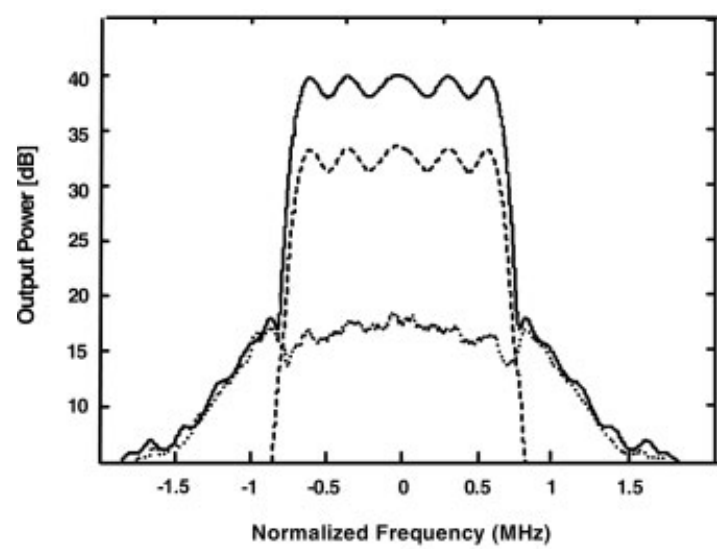

a)

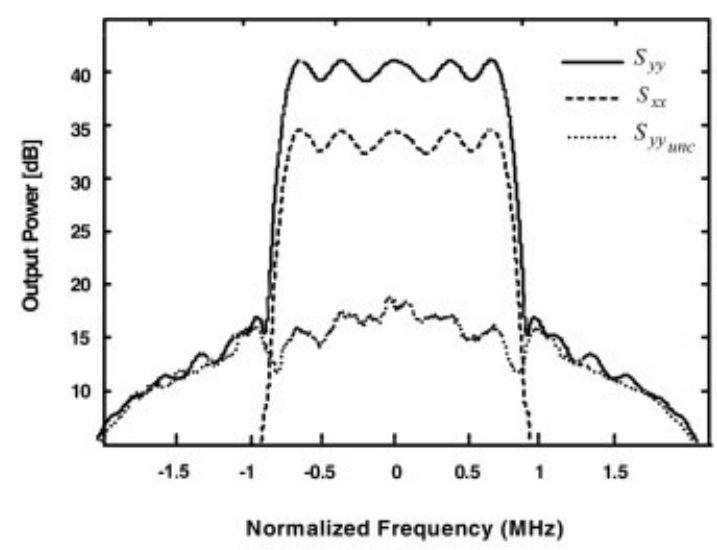

c)

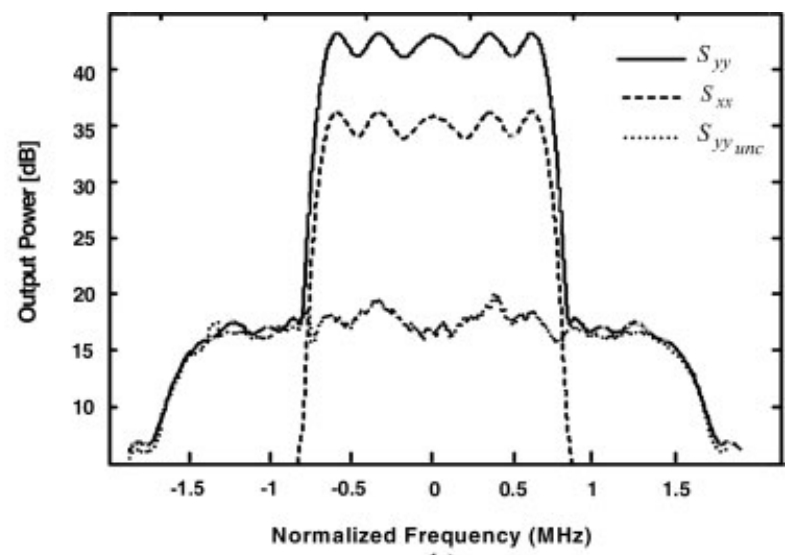

b)

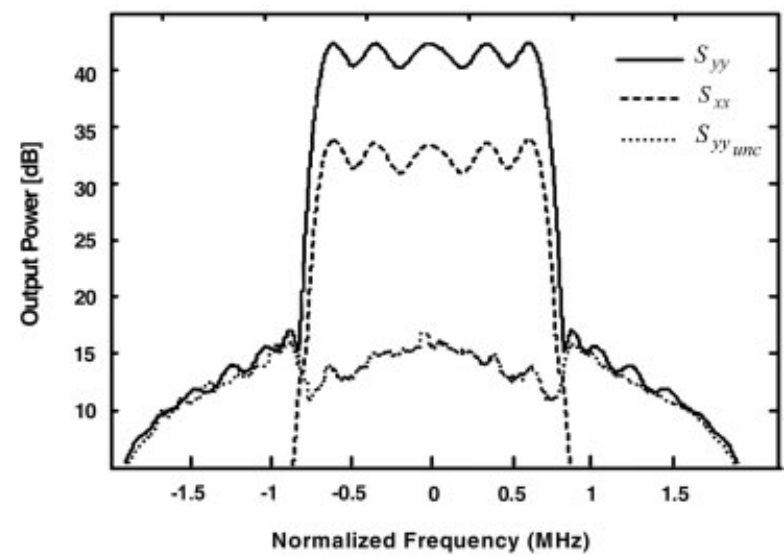

d)

Figure 6. Total uncorrelated memory spectrum mask for (a) 2/1 BW; (b) $1 / 1 \mathrm{BW}$; (c) $1 / 4 \mathrm{BW}$; (d) memoryless.

Figure 6 for three different filters, and for the memoryless case. There, it is visible that the memory effects will impress a new spectrum characteristic on both the cochannel and the spectral regrowth.

The differences in cochannel uncorrelated distortion shapes can be attributed to a dramatic change of the baseband filter response which not only changes the spectrum, but also the statistical behavior of the pure second-order baseband time domain signal.

From Figure 6, it is clearly seen that the uncorrelated cochannel distortion is higher for each of the three situations with memory, compared with the memoryless case. Moreover it is also seen that the very narrow band filter and the very wide band filter, have a similar response, since both of them are imposing a low value of uncorrelated distortion arising from the base band path.

The narrow band filter has little impact on the distortion, and the large bandwidth filter is similar to a memoryless case, where all the baseband response is considered, similar as if we have $x(t)^{2} x(t)=x(t)^{3}$.

The mid-band filter is in fact the one that imposes a strong impact on the nonlinear output distortion, since as can be seen, not only the cochannel uncorrelated distortion has change its shape, but also the spectral regrowth as raised significantly compared with the other cases. These results are in accordance to what was expected from the baseband analysis.

\section{EXPERIMENTAL RESULTS}

When simulating this transmitter, the nonlinear distortion components are readily available, as we can separate each branch on the model. Thus, the study of uncorrelated distortion is straightforward as it is not corrupted by the linear components, as was stated on section II.

Nevertheless in real measurement data it is not possible to separate it, so we have to apply the measurement procedure previously presented.

To evaluate cochannel distortion in real wireless transmitters we have generated an IS-95 CDMA reverse channel as the excitation signal and apply it 


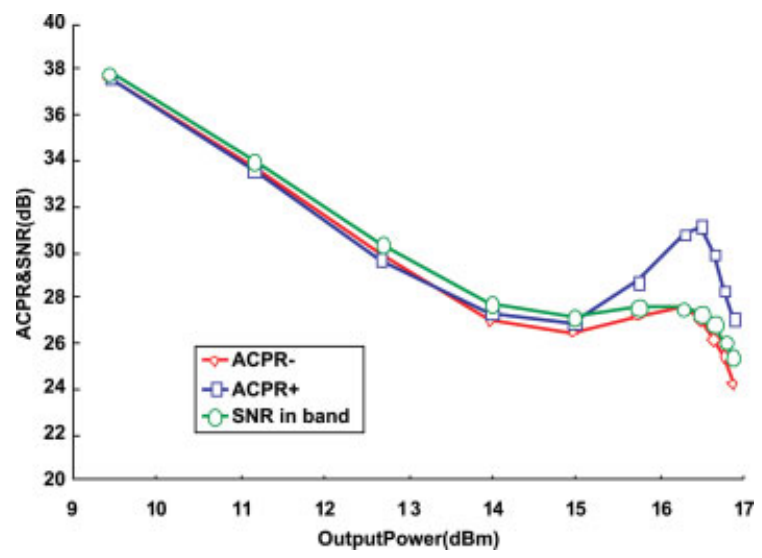

Figure 7. IS-95 excitation passed through a PA nonlinearity presenting memory. [Color figure can be viewed in the online issue, which is available at www.interscience. wiley.com.]

to a PA presenting memory. Figure 7 presents the results for an input power sweep both for the cochannel and adjacent channel distortions.

An interesting observation that can be gathered from this graph is that the SNDR also presents a large signal sweet spot, despite it follows the worst behavior of the ACPR values as expected.

Therefore, the uncorrelated cochannel distortion is impacted very similarly as the ACPR, but since the cochannel distortion is integrated over the signal bandwidth, the overall degradation is dominated by the increase in distortion caused by the memory effect, as seen in section III.

We have furthered try to model this PA with a memoryless behavioral model based on AM-AM and AM-PM responses, and compared it to the real measured values as shown in Figure 8. The memoryless model indicates a consistent increase in cochannel

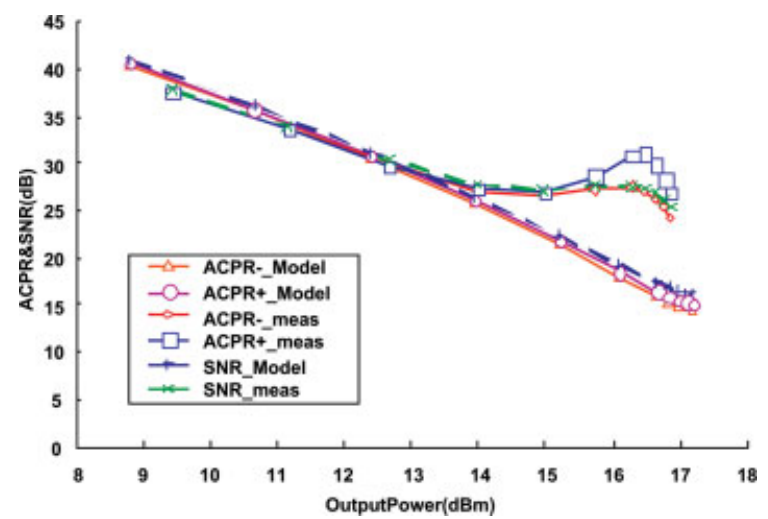

Figure 8. Comparison of ACPR and SNDR measured and predicted by a memoryless model of the amplifier. [Color figure can be viewed in the online issue, which is available at www.interscience.wiley.com.]

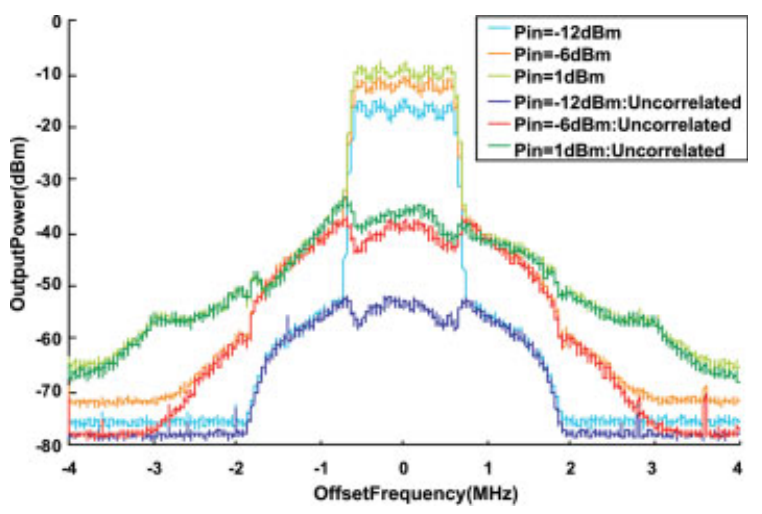

Figure 9. Output spectrum of the IS-95 excitation passed through a PA nonlinearity presenting memory. [Color figure can be viewed in the online issue, which is available at www.interscience.wiley.com.]

and adjacent channel distortion compared to the measurements, and at large signal no sweet spot is seen. We have also plotted the output spectrum in Figure 9, where a clear impact is visible at the large signal sweet spot both at the cochannel and adjacent channel distortion.

This states that in this case the usual used AM/ $\mathrm{AM}$ and $\mathrm{AM} / \mathrm{PM}$ model could not be used to predict cochannel distortion anymore, and thus an improved behavioral model should be used for describing the transmitter electrical behavior.

We have then selected two different power inputs, one presenting a high value of ACPR asymmetry, and thus of memory effects, and another one presenting a similar up and lower distortion patterns. Figures 10 and 11, presents the seek results, for the case of the PA presenting memory and for a memoryless PA.

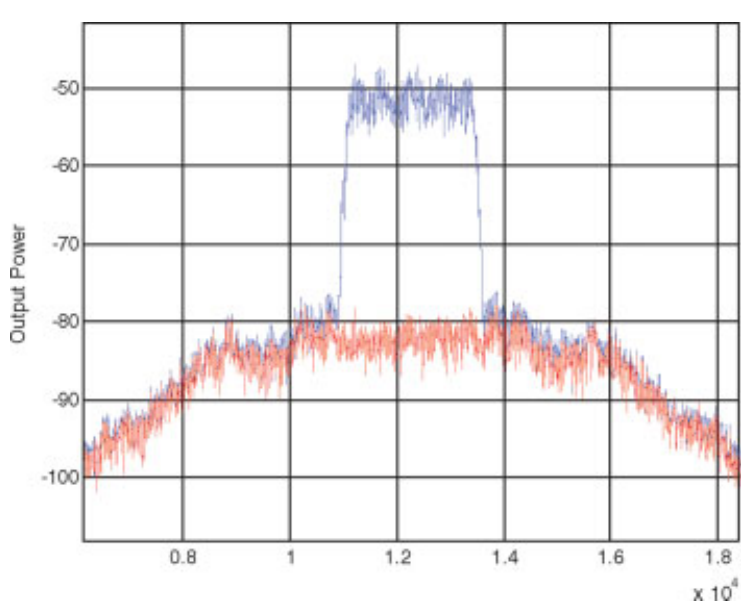

Figure 10. IS-95 excitation passed through a PA nonlinearity presenting memory. [Color figure can be viewed in the online issue, which is available at www.interscience. wiley.com.] 


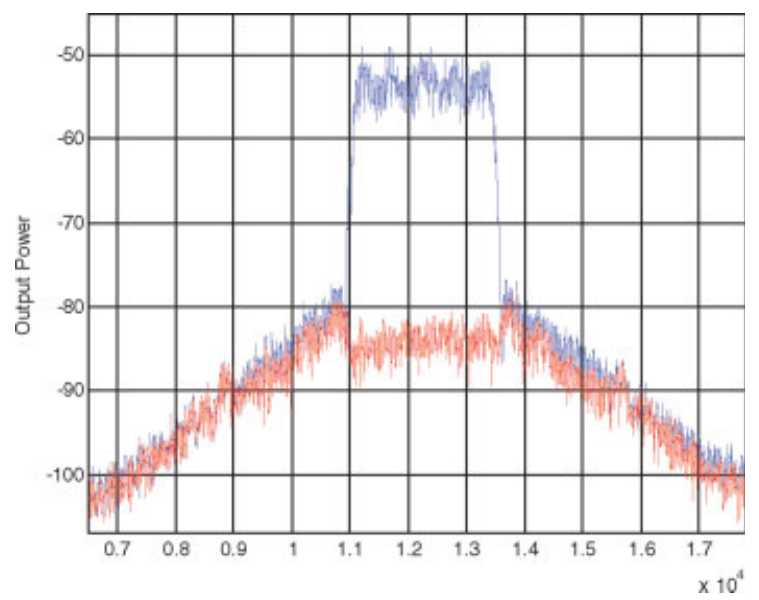

Figure 11. IS-95 excitation passed through a memoryless PA nonlinearity. [Color figure can be viewed in the online issue, which is available at www.interscience.wiley.com.]

As was expected the memory effect case presents a clear rise in the cochannel distortion, degrading in that respect the SNDR, and a rise in spectral regrowth with a plateau shape as was predicted by simulations.

\section{CONCLUSIONS}

In this article, a carefully study of the cochannel distortion in PA was made, and a new measurement bench for measuring the cochannel distortion on PA presenting memory was also presented.

We observe that the memory effects have a strong impact on the cochannel distortion in wireless systems, which can compromise the system performance, and thus the SNDR improvement/degradation.

In fact the results now presented impose a different view on cochannel distortion in O-QPSK/CDMA based signals when compared to ideal memoryless devices.

The experimental results state that the large signal SNDR sweet spot is also visible on IS-95 systems, even in PA's presenting memory effects, which is an important aspect for RF design engineers.

\section{ACKNOWLEDGMENTS}

This work was partially supported by the EU under the Network of Excellence, TARGET contract IS-1-507893NoE project Musilage and ColteMepai.

\section{REFERENCES}

1. K.M. Gharaibeh, K. Gard, and M. Steer, Accurate estimation of digital communication system metrics-SNR, EVM and in a nonlinear amplifier environment, ARTFG Microwave Measurements Conference, Honolulu, Hawaii, 2004.

2. P.M. Lavrador, de N.B. Carvalho, and J.C. Pedro, Evaluation of signal-to-noise and distortion ratio degradation in nonlinear systems, IEEE Trans Microwave Theory Tech 52 (2004), 813-822.

3. V. Aparin, Analysis of CDMA signal spectral regrowth and waveform quality, IEEE Trans Microwave Theory Tech 49 (2001), 2306-2314.

4. K.G. Gard, H.M. Gutierrez, and M.B. Steer, Characterization of spectral regrowth in microwave amplifiers based on the nonlinear transformation of a complex Gaussian process, IEEE Trans Microwave Theory Tech 47 (1999), 1059-1069.

5. K.M. Gharaibeh, K. Gard, and M.B. Steer, The impact of nonlinear amplification on the performance of CDMA systems, IEEE Radio Wireless Conf (2004), 83-86.

6. R.E. Santos, N.B. Carvalho, and K. Gard, The impact of long term memory effects in wireless QPSK modulated signals, International Microwave Symposium, Honolulu, 2007, pp. 961-964.

7. J.C. Pedro and N.B. Carvalho, Intermodulation distortion in microwave and wireless circuits, Artech House, Norwood MA, 2003.

8. J.C. Pedro and N. Borges de Carvalho, Evaluating cochannel distortion ratio in microwave power amplifiers, IEEE Trans Microwave Theory Tech 49 (Part 1) (2001), 1777-1784.

9. H. Ku, W. Woo, and J.S. Kenney, Carrier-to-interference ratio prediction of nonlinear RF devices, Microwave J 44 (2001), 154-164.

10. J. Bendat, Random data: Analysis and measurement procedures, 3rd ed. New York, Wiley, 2000.

11. J.P. Martins, P.M. Cabral, N. Borges Carvalho, and J.C. Pedro, A metric for the quantification of memory effects in power amplifiers, IEEE Trans Microwave Theory Tech 54 (Part 2) (2006), 4432-4439.

12. J.P. Martins, N.B. Carvalho, and J.C. Pedro, Intermodulation distortion of third-order nonlinear systems with memory under multisine excitations, IEEE Trans Microwave Theory Tech 55 (Part 2) (2007), 12641271.

13. A. Walker, M. Steer, and K. Gard, Capturing asymmetry in distortion of an RF system using a multislice behavioral model, IEEE Microwave Wireless Comp Lett 16 (2006), 212-214. 


\section{BIOGRAPHIES}

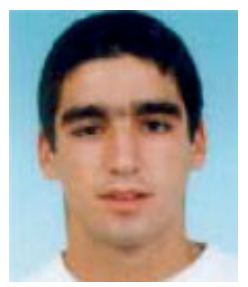

Rui Estanqueiro Santos was born in Torres Novas, in 1982. He received the diploma and master degrees in Electronics and Telecommunications Engineering from the University of Aveiro, Aveiro, Portugal in 2005 and 2007 respectively. Currently is a wireless planning engineer at SONAE.COM.

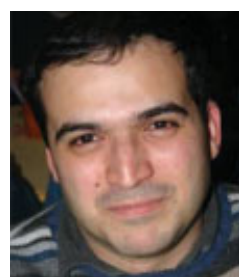

Nuno Borges Carvalho was born in Luanda, in 1972. He received the diploma and doctoral degrees in Electronics and Telecommunications Engineering from the University of Aveiro, Aveiro, Portugal in 1995 and 2000, respectively. Currently, he is an Associate Professor at the University of Aveiro and a senior research scientist at the Telecommunications Institute. He has worked as a scientist researcher at the Telecommunications Institute, and was engaged to different projects on nonlinear CAD and circuits. His main research interests include CAD for nonlinear circuits and design of RF-microwave power amplifiers. Dr. Carvalho is a member of the Portuguese Engineering Association, an URSI member and an IEEE member. He was the recipient of the 1995 University of Aveiro and the Portuguese Engineering Association Prize for the best 1995 student at the Universidade de Aveiro, the 1998 Student Paper Competition (third place) presented at the IEEE International Microwave Symposium and the 2000 IEE Measurement Prize. He has been a reviewer for several magazines and is a member of the IEEE Microwave Theory and Techniques Society MTT-11 Technical Committee. Dr. Carvalho is coauthor of the book "Intermodulation in Microwave and Wireless Circuits" from Artech House, 2003

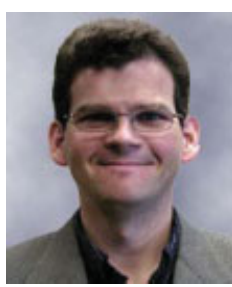

Kevin G. Gard received the B.S. and M.S. degrees in electrical engineering from North Carolina State University, Raleigh, in 1994 and 1995, respectively, and the Ph.D. degree in electrical engineering from the University of California at San Diego, La Jolla, in 2003. He is currently the William J. Pratt Assistant Professor with the Electrical and Computer Engineering Department, North Carolina State University. From 1996 to 2003, he was with Qualcomm Inc., San Diego, CA, where he was a Staff Engineer and Manager responsible for the design and development of RF integrated circuits (RFICs) for code-division multiple-access (CDMA) wireless products. He has designed SiGe BiCMOS, Si BiCMOS, and GaAs metal-semiconductor field-effect transistor (MESFET) integrated circuits for cellular and personal communication systems (PCSs) CDMA, wideband code-division multipleaccess (WCDMA), and AMPS transmitter applications. His research interests are in the areas of integrated circuit design for wireless applications and analysis and modeling of nonlinear microwave circuits with digitally modulated signals. Dr. Gard is a member of the IEEE Microwave Theory and Techniques and Solid-State Circuits Societies, Sigma Xi, Eta Kappa Nu, and Tau Beta Pi. 